\title{
On ramified covers of the projective plane II: Generalizing Segre's theory
}

\author{
Received March 11, 2010
}

\begin{abstract}
The classical Segre theory gives a necessary and sufficient condition for a plane curve to be a branch curve of a (generic) projection of a smooth surface in $\mathbb{P}^{3}$. We generalize this result for smooth surfaces in a projective space of any dimension in the following way: given two plane curves, $B$ and $E$, we give a necessary and sufficient condition for $B$ to be the branch curve of a surface $X$ in $\mathbb{P}^{N}$ and $E$ to be the image of the double curve of a $\mathbb{P}^{3}$-model of $X$.

In the classical Segre theory, a plane curve $B$ is a branch curve of a smooth surface in $\mathbb{P}^{3}$ iff its 0 -cycle of singularities is special with respect to a linear system of plane curves of particular degree. Here we prove that $B$ is a branch curve of a surface in $\mathbb{P}^{N}$ iff (part of) the cycle of singularities of the union of $B$ and $E$ is special with respect to the linear system of plane curves of a particular low degree. In particular, given just a curve $B$, we provide some necessary conditions for $B$ to be a branch curve of a smooth surface in $\mathbb{P}^{N}$.
\end{abstract}

\section{Introduction}

Let $X$ be a non-singular algebraic surface of degree $v$ in $\mathbb{P}^{N}$ (we work over an algebraically closed field of characteristic 0 ). Choosing a generic linear subspace $W$ of codimension 3 in $\mathbb{P}^{N}$ and considering the projection of $\mathbb{P}^{N}$ to a plane with center $W$, we get a ramified cover $\pi: X \rightarrow \mathbb{P}^{2}$. Let $B$ be the branch curve of $\pi$; it is known to be an irreducible nodal-cuspidal curve. Such branch curves are special among all nodal-cuspidal curves with the same type of singularities; for example, in the simplest and the most well-known case of a cubic surface in $\mathbb{P}^{3}$, the branch curve $B$, which is a plane sextic with six cusps, is special since all of its six cusps lie on a conic (see Segre and Zariski [24], [27]). Segre studied the question of whether the singularities of $B$ form a special configuration of points in the plane for a surface of any degree in $\mathbb{P}^{3}$, as in the case of a surface of degree 3 , and found a generalization of this statement. Moreover, he proved that this property uniquely characterizes branch curves and that one can reconstruct the surface from its branch curve.

M. Friedman, M. Teicher: Department of Mathematics, Bar-Ilan University, 52900 Ramat Gan, Israel; e-mail: fridmam@macs.biu.ac.il, teicher@macs.biu.ac.il

R. Lehman: Department of Mathematics, Hebrew University, Givat Ram, 91904 Jerusalem, Israel; e-mail: rlehman@math.huji.ac.il

M. Leyenson: e-mail: leyenson@gmail.com 
More specifically, let $S$ be a smooth surface in $\mathbb{P}^{3}$; its branch curve is known to be of degree $v(v-1)$, have $c(v)=v(v-1)(v-2)$ cusps and $n(v)=\frac{1}{2} v(v-1)(v-2)(v-3)$ nodes. Let $a(v)=(v-1)(v-2)$. Segre proved that a nodal-cuspidal plane curve $B$ of degree $v(v-1)$ with $n(v)$ nodes and $c(v)$ cusps is a branch curve of a generic projection of a smooth surface of degree $v \geq 3$ in $\mathbb{P}^{3}$ if and only if there are two adjoint curves of degrees $a(v)$ and $a(v)+1$ passing through the 0 -cycle of singularities of $B$ and having separated tangents and these singularities. In particular, the 0-cycle of singularities of $B$ is special. (See [24] for Segre's original proof, but also [5], [19] and [8] for recent surveys.)

In light of this necessary and sufficient condition for a curve to be a branch curve of a smooth surface in $\mathbb{P}^{3}$, it was natural for Chisini [3] to conjecture that a generic ramified cover of the plane $\mathbb{P}^{2}$ of degree at least 5 is uniquely determined by its branch curve. This conjecture was proved by Kulikov (in [17] for ramified covers of degree at least 11, and then in [20] for generic linear projections of surfaces other than Veronese), but his proof is not constructive.

Given a smooth surface $X$ in $\mathbb{P}^{N}, N>3$, one can decompose any projection $\pi$ : $X \rightarrow \mathbb{P}^{2}$ as a composition of a generic projection $X \rightarrow \mathbb{P}^{3}$ and a projection $\mathbb{P}^{3} \rightarrow \mathbb{P}^{2}$. Let $S$ be the image of $X$ in $\mathbb{P}^{3}$. It is known that $S$ is a surface with ordinary singularities, i.e., it has a double curve as its singular locus. (Note that the class of surfaces with ordinary singularities in $\mathbb{P}^{3}$ is broader than the class of images of generic linear projections of smooth surfaces in $\mathbb{P}^{N}$; in particular, if $E$ has multiple components then $X$ is not a generic linear projection of a smooth surface.)

One can check that the branch curve of the projection of $S$ to $\mathbb{P}^{2}$ is a union of the branch curve $B$ of the projection $X \rightarrow \mathbb{P}^{2}$ and the image $2 E$ of the double curve $2 E^{*}$ with the corresponding double (scheme) structure. (We sometimes call $B$ a pure branch curve for the projection of $S$ to $\mathbb{P}^{2}$.)

One possible direction toward generalizing Segre's theory for the smooth surface $X$ in $\mathbb{P}^{N}$ is to consider its image $S$ in $\mathbb{P}^{3}$ and ask the same questions for the total branch curve, i.e., to study the configuration of the singularities of the branch curve $B \cup 2 E$ on the plane. Slightly more generally, we can consider any surface $S$ in $\mathbb{P}^{3}$ with ordinary singularities (not necessarily a projection of a surface from $\mathbb{P}^{N}$ ) projected further to $\mathbb{P}^{2}$, and try to generalize Segre's theory to this class of surfaces.

We ask the following questions: What are the special properties of the pure branch curve $B$, or the total branch curve $B \cup 2 E$ ? Can one construct a singular surface $S$ in $\mathbb{P}^{3}$, given two plane curves, a nodal-cuspidal curve $B$ and a double curve $2 E$, where $E$ possibly has triple points and nodes as singularities, and two adjoint curves (to $B \cup E$ ) such that the total branch curve of $S$ is $B \cup 2 E$ ?

In this paper we give answers to these questions. We prove that the branch curve $B$ has two adjoint curves, which also pass through some of the singularities of $B \cup E$, such that one can (re)construct a surface $S$ with ordinary singularities given $B, E$, and these two adjoint curves. Thus, there is a 0-cycle of points on $B$ consisting of cusps and the nodes of $B$ and two other special sets of points (called the "vertical" points and the "new" intersection points of $B$ and $E$ ), which is a special 0-cycle on the plane. 
As a consequence, we have a constructive proof of the analogue of Chisini's conjecture for surfaces in $\mathbb{P}^{3}$ with ordinary singularities. That is, any such surface in $\mathbb{P}^{3}$ is determined uniquely and constructively by its total branch curve in $\mathbb{P}^{2}$.

This paper is organized as follows. In Section 2 we give the necessary background regarding surfaces in $\mathbb{P}^{3}$. Section 3 proves that if $B$ is the pure branch curve of a singular surface $S \subseteq \mathbb{P}^{3}$, then $B$ (respectively, $B \cup E$ ) has two adjoint curves, and in particular, there is a special 0-cycle on $B$ that consists of the singularities of $B$ (and the intersection of $B$ and $E$ ). In Section 4 we prove the converse, i.e., the sufficiency of these two adjoint curves to construct a surface $S$ with a given (total) branch curve $B \cup 2 E$. We conclude the paper with a few examples in Section 5.

\section{Surfaces in $\mathbb{P}^{3}$}

Let $X$ be a smooth projective surface in $\mathbb{P}^{N}$. Projecting $X$ to $\mathbb{P}^{2}$ by a generic linear projection, we know that the branch curve $B$ is a nodal-cuspidal curve (see [4] for a modern proof). However, $X$ can be first projected onto $\mathbb{P}^{3}$, where its image has a double curve. In this section we review the relations between the ramification curve, the double curve and their images in $\mathbb{P}^{2}$.

It is classical that any smooth projective surface $X$ can be embedded into $\mathbb{P}^{5}$ as a smooth surface. However, when projecting generically from $\mathbb{P}^{5}$ to $\mathbb{P}^{3}$, the image $S \subset \mathbb{P}^{3}$ of $X$ is a singular surface with so-called "ordinary singularities" (this is, of course, also true for smooth surfaces in $\mathbb{P}^{4}$ ). The singular locus of $S$ is well known (see, e.g., [12]): it consists of a double curve whose only singularities are some triple and pinch points. Explicitly, in terms of a local holomorphic coordinate system $(x, y, z)$, a local model of the surface $S$ is as follows:

- in a neighborhood of a smooth point of the double curve, $S=\{x y=0\}$;

- in a neighborhood of a triple point, $S=\{x y z=0\}$;

- in a neighborhood of a pinch point, $S=\left\{x^{2}-y z^{2}=0\right\}$.

Notation 2.1. Scheme-theoretically, the double curve $F^{*}$ of $S$ is given as the annihilator of the pushforward of the sheaf $\Omega_{X / S}^{1}$ with respect to the map $f: X \rightarrow S$. The reduced closed subscheme structure of the double curve of $S$, which is denoted by $E^{*}$, is the support of $F^{*}$.

Remark 2.2. In $A_{1}(S)$, we have $\left[F^{*}\right]=2\left[E^{*}\right]$, where $\left[F^{*}\right]$ is the Weil divisor associated with the Cartier divisor $2\left[E^{*}\right]$.

It is known that $E^{*}$ is irreducible unless $X=V_{2}$, the Veronese embedding of $\mathbb{P}^{2}$ in $\mathbb{P}^{5}$, where in this case the (reduced) double curve $E^{*}$ of $f(X)=S$ is a union of three non-coplanar lines meeting in one point. See [21, Theorem 3] and [6]. Note, however, that there are surfaces in $\mathbb{P}^{3}$ with ordinary singularities such that their double curve is reducible, i.e., they are not generic projections of a smooth surface in $\mathbb{P}^{N}, N>3$. For example, there is a degree 4 surface in $\mathbb{P}^{3}$ with two skew lines as a double curve (see e.g. $[12$, p. 630]). 
Assume now that we are given a degree $v$ surface $S=\{f=0\} \subset \mathbb{P}^{3}=\mathbb{P}(V)$ with ordinary singularities and a point $O$ not on $S$. We want to emphasize that $S$ is not necessarily the projection of a smooth surface, and its double curve may be reducible. Consider the projection map $\pi: S \rightarrow \mathbb{P}\left(V / l_{O}\right) \simeq \mathbb{P}^{2}$ (where $l_{O}$ is the line in $V$ corresponding to the point $O$ in $\mathbb{P}(V)$ ), and define the polar surface of $S$ with respect to $O$ as

$$
S_{O}^{\prime}=\left\{\sum O_{i} \frac{\partial f}{\partial x_{i}}=0\right\} .
$$

The ramification curve $B_{\text {Total }}^{*}$ of the projection is defined as the scheme-theoretic intersection of $S$ and the polar surface $S_{O}^{\prime}$. Note that $B_{\text {Total }}^{*}=S \cap S_{O}^{\prime}$ is the annihilator of the sheaf $\Omega_{S / \mathbb{P}^{2}}$.

One can now see that $B_{\text {Total }}^{*}$ can be decomposed (scheme-theoretically and in $Z_{1}(S)$ ) as

$$
\left[B_{\text {Total }}^{*}\right]=\left[B^{*}\right]+\left[F^{*}\right] .
$$

Note that the set-theoretic intersection $B^{*}$ of $S_{O}^{\prime}$ with the smooth locus of $S$ is the set of smooth points $p$ on $S$ such that the tangent plane $T_{p}(S)$ contains $O$. Scheme-theoretically, $B^{*}$ is the support of the kernel sheaf of the canonical map $\Omega_{S / \mathbb{P}^{2}}^{1} \rightarrow i_{*} i^{*} \Omega_{S / \mathbb{P}^{2}}^{1} \rightarrow 0$, where $i$ is the embedding of $F^{*}$ into $S$ (where this map is associated to the left adjointness of $i_{*}$ ). For a different scheme-theoretic description of $E^{*}$ and $B^{*}$, see [22, Section 2].

Remark 2.3. For a smooth surface $X \subset \mathbb{P}^{N}, N \geq 3$, any generic projection $X \subset$ $\mathbb{P}^{N} \rightarrow \mathbb{P}^{2}$ can be factored as a composition of projections $X \subset \mathbb{P}^{N} \rightarrow \mathbb{P}^{3} \rightarrow \mathbb{P}^{2}$ such that, letting $S=\operatorname{Im}(X) \subset \mathbb{P}^{3}$, the projection $S \rightarrow \mathbb{P}^{2}$ is also generic. If we first project $X$ to $\mathbb{P}^{3}$, and then from $\mathbb{P}^{3}$ to $\mathbb{P}^{2}$, we get an extra component of the branch curve: if $B^{*} \subset \mathbb{P}^{2}$ is the ramification curve of the direct projection $X \subset \mathbb{P}^{N} \rightarrow \mathbb{P}^{2}$ and $F^{*} \subset \mathbb{P}^{3}$ is the double curve, then, in $Z_{1}\left(\mathbb{P}^{2}\right)$,

$$
\left[B_{\text {Total }}\right]=[B]+[F],
$$

where $B_{\text {Total }}, B$ and $F$ are the scheme-theoretic images of $B_{\text {Total }}^{*}, B^{*}$ and $F^{*}$, respectively. Of course, the direct projection of $X$ from $\mathbb{P}^{N}$ to $\mathbb{P}^{2}$ "does not know" about the double curve of $S$ in $\mathbb{P}^{3}$. As before, in terms of cycle classes, group $A_{1}\left(\mathbb{P}^{2}\right)$, we have $[F]=2[E]$.

From now on, let $S$ be a surface in $\mathbb{P}^{3}$ with ordinary singularities.

Notation 2.4. Let $u$ be the number of components of $E^{*}$ and let $E_{i}^{*}$ be these components. Set

$e=\operatorname{deg} E^{*}, e_{i}=\operatorname{deg} E_{i}^{*}, v=\operatorname{deg} S, d=\operatorname{deg} B^{*}=v(v-1)-2 e=\operatorname{deg} B^{*}-2 \operatorname{deg} E^{*}$.

Remark 2.5. A generic hyperplane section $S \cap H$ of $S$ is a plane curve of degree $v$ with $e$ nodes at the points of $E^{*} \cap H$, so

$$
0 \leq e \leq \frac{(v-1)(v-2)}{2}
$$


since the number of nodes of a plane curve cannot exceed its arithmetic genus. It follows that the pair $(v, d)$ satisfies the relation

$$
2(v-1) \leq d \leq v(v-1) .
$$

Note that for a given $d$ there are only a finite number of possible values of $v$ such that a plane curve $C$ of degree $d$ can be a pure branch curve of a degree $v$ surface in $\mathbb{P}^{3}$ with ordinary singularities. We discussed the geography of this domain in our previous paper [8].

Definition 2.6. We define

$$
Q_{\text {Total }}^{*}=B_{\text {Total }}^{*} \cap S_{O}^{\prime \prime}
$$

to be the scheme-theoretic intersection of $B_{\text {Total }}^{*}$ and the second polar surface $S_{O}^{\prime \prime}$, i.e., the intersection of $S, S_{O}^{\prime}$ and $S_{O}^{\prime \prime}$. For a smooth surface, the points $Q_{\text {Total }}^{*}$ are the preimages of the cusps of the branch curve $B$ (see e.g. [25]). However, for a singular surface $S$, not all the points of $Q_{\text {Total }}^{*}$ form cusps on the branch curve. (See Lemma 2.9.)

We denote by $Q^{*}$ the smooth points of $B^{*}$ such that their images $\pi\left(Q^{*}\right)$ are the cusps of $B$.

Notation 2.7. Denote by $V_{i}^{*} \in E_{i}^{*}$ the points such that the tangent plane to $S$ at $V_{i}^{*}$ contains the center of projection $O$. We call these points vertical points, as we think of $O$ as being "high-above" (or points of immersion, in [25, Chapter VII, Section 3]). Let $V^{*}=\bigcup V_{i}^{*}$.

Denote by $T^{*}$ the set of triple points of $E^{*}=\bigcup E_{i}^{*}$, and by $t$ the number of these points. Let $\bigcup_{j=1}^{t} \tau_{i j}^{*}$ be the set of triple points of $E_{i}^{*}$ (indexed by $j$ ) with their multiplicities on the component $E_{i}$ (see [12, p. 622]). Note that $\left|\sum_{i} \tau_{i j}^{*}\right|=3$ and $\left|\sum_{i, j} \tau_{i j}^{*}\right|=3 t$. We introduce this notation as we have to take into account the fact that there can be different types of spatial triple points, arising from the fact that a triple point can be composed from several components of the branch curve. In the figure below we picture the different arrangements, with the corresponding $\tau$ 's.

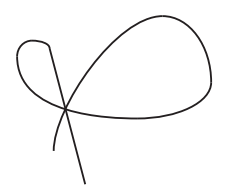

$\tau=3$

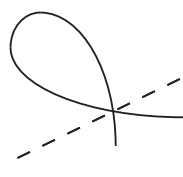

$\tau_{1}=2, \tau_{2}=1$

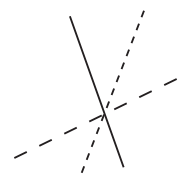

$\tau_{1}=1, \tau_{2}=1, \tau_{3}=1$

Fig. 1

Let Pinch ${ }_{i}^{*}$ be the set of pinch points of $E_{i}^{*}$ and let $p_{i}$ be the number of these points. Denote Pinch* $=\bigcup_{i}$ Pinch $_{i}^{*}$ and $p=\sum_{i} p_{i}$.

For any curve $C \subset \mathbb{P}^{N}$ let $h_{C}$ be the Cartier class of the hyperplane section of $C$.

Remark 2.8. Note that the number $p$ of pinch points is always even (see [10] or [21, Theorem 3(4)]) and it is 0 only when $S$ is a smooth surface in $\mathbb{P}^{3}$. 
The following lemma is proved in [25, Chapter IX, Sections 3.1, 3.2].

Lemma 2.9. (1) $Q_{\text {Total }}^{*}=S_{O}^{\prime \prime} \cap B_{\text {Total }}^{*}$ can be decomposed as

$$
\left[Q_{\text {Total }}^{*}\right]=\left[Q^{*}\right]+\left[S_{O}^{\prime \prime} \cap F^{*}\right] .
$$

Note that the images of points of $S_{O}^{\prime \prime} \cap E^{*}$ under the projection are smooth points of $B$.

(2) Points in $B^{*} \cap E^{*}$ do not project to nodes or cusps of the branch curve, i.e., their images are smooth points on B. Moreover, scheme-theoretically (or in $Z_{0}\left(B^{*}\right)$, the group of 0-cycles),

$$
B^{*} \cap E^{*}=\operatorname{Pinch}^{*} \cup V^{*}, \quad B^{*} \cap E_{i}^{*}=\operatorname{Pinch}_{i}^{*} \cup V_{i}^{*} .
$$

(3) In $Z_{0}\left(E^{*}\right), S_{O}^{\prime \prime} \cap E^{*}$ can be decomposed as

$$
\left[S_{O}^{\prime \prime} \cap E^{*}\right]=\left[V^{*}\right]+3\left[T^{*}\right]
$$

and in $Z_{0}\left(E_{i}^{*}\right)$ the equation is

$$
\left[S_{O}^{\prime \prime} \cap E_{i}^{*}\right]=\left[V_{i}^{*}\right]+\sum_{j}\left[\tau_{i j}^{*}\right] .
$$

In $Z_{0}\left(B^{*}\right), S_{O}^{\prime \prime} \cap B^{*}$ can be decomposed as

$$
\left[S_{O}^{\prime \prime} \cap B^{*}\right]=\left[V^{*}\right]+\left[Q^{*}\right] .
$$

We cite here from [12, p. 628] the computation of the number of pinch points on $E_{i}^{*}$.

Lemma 2.10.

$$
\begin{aligned}
p_{i} & =2(v-4) e_{i}-2 \sum_{j}\left|\tau_{i j}\right|-4\left(g_{i}-1\right), \\
p & =2(v-4) e-6 t-4(g-u),
\end{aligned}
$$

where $g=\sum g_{i}$ is the sum of the arithmetic genera of $E_{i}^{*}$, and $u$ is the number of components of $E^{*}$.

Proof (see [12, p. 628]). By Riemann-Hurwitz and adjunction on the blow up of $S$ with respect to the triple points and the double curve.

Remark 2.11. In the case when $S$ is a generic projection of a smooth surface, if $S$ is not the image of the Veronese surface $V_{2}$, then $u=1$. When $S$ is the image of $V_{2}, u=3$, $p=6$ and on each double line there are two pinch points $\left(\left|\operatorname{Pinch}_{i}\right|=2,1 \leq i \leq 3\right.$; see e.g. [25]).

Remark 2.12. In [12, p. 624] the Chern classes $c_{1}^{2}, c_{2}$ of $S$ are expressed in terms of $v$, $e, t$ and $g-u$ :

$$
\begin{aligned}
& c_{1}^{2}=v(v-4)^{2}-5 v e+24 e+4(g-u)+9 t, \\
& c_{2}=v^{2}(v-4)+6 v+24 e-7 v e+8(g-u)+15 t .
\end{aligned}
$$




\section{From ramification curves to adjoint curves}

\subsection{The pure branch curve}

When projecting a surface $S \subset \mathbb{P}^{3}=\mathbb{P}(V)$ to the plane $\Pi=\mathbb{P}\left(V / \ell_{O}\right)$ from a generic point $O \in \mathbb{P}^{3}, O \notin S\left(O\right.$ is the projectivization of a 1-dimensional space $\left.\ell_{O} \subset V\right)$, the (pure) ramification curve $B^{*}$ and the reduced double curve $E^{*}$ project to two singular plane curves $B$ and $E$, respectively. Denote the projection map by $\pi: S \rightarrow \Pi$.

The singular points of $B$ are nodes, denoted by $P$, and cusps, denoted by $Q$, while the curve $E$ has as singularities nodes (arising from the projection $E^{*}$ to $E$ ), denoted by Node, and triple points, denoted by $T$.

We recall (from Lemma 2.9) that $B^{*}$ and $E_{i}^{*}$ intersect at the pinch points Pinch ${ }_{i}^{*}$ and at the vertical points $V_{i}^{*}$.

Let Pinch $\left(\right.$ resp. $V_{i}$ ) be the image of Pinch ${ }_{i}^{*}\left(\right.$ resp. $V_{i}^{*}$ ) under the projection $S \rightarrow \Pi$. Thus, set-theoretically, $B$ and $E_{i}$ intersect at the images of the pinch points $\mathrm{Pinch}_{i}$ and at the vertical points $V_{i}$, and also potentially at some new points $N_{i}$ :

$$
B \cap E_{i}=\operatorname{Pinch}_{i} \cup V_{i} \cup N_{i} .
$$

This $N_{i}$ is the intersection points of $\Pi$ with bisecants to $B^{*}$ passing through $O$ and intersecting both $B^{*}$ and $E_{i}^{*}$ (at two distinct points).

Let Node ${ }_{i}$ be the set of nodes of $E_{i}$ that do not coincide with any of the triple points of $E$. Note that the sum of the lengths of 0 -cycles \#( $\left(\mathrm{Node}_{i}\right)$ may be strictly less than \#(Node), as some nodes of $E$ may arise from lines through $O$ intersecting two different components of $E^{*}, E_{i}^{*}$ and $E_{j}^{*}$.
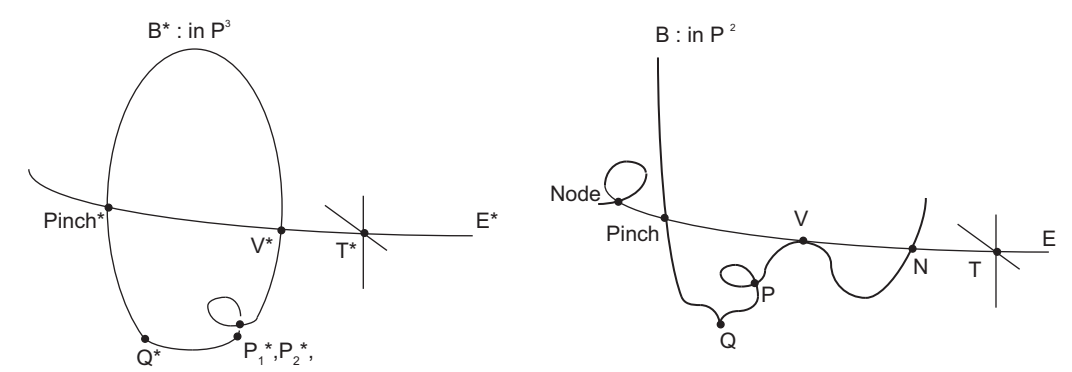

Fig. 2. The ramification curve and the branch curve.

We now prove some relations in the Chow groups $A_{0}(B)$ and $A_{0}\left(E_{i}\right)$.

Lemma 3.1.

$$
\begin{aligned}
& {\left[\text { Pinch }_{i}\right]+2\left[V_{i}\right]+\left[N_{i}\right]=e_{i} h_{B} \quad \text { in } A_{0}(B) \text {, }} \\
& {\left[\operatorname{Pinch}_{i}\right]+2\left[V_{i}\right]+\left[N_{i}\right]=(v(v-1)-2 e) h_{E_{i}} \text { in } A_{0}\left(E_{i}\right) \text {. }}
\end{aligned}
$$

Proof. This is easily verified by a local computation for $B \cap E_{i}$. The curves $B$ and $E_{i}$ intersect transversely at $\mathrm{Pinch}_{i}$ and $N_{i}$, and are simply tangent at the vertical points. 
Note that the version of this equation on $E$ (and all the other equations that follow) is induced by summing over $i$.

Definition 3.2. Let $C=\{f=0\}$ be a reduced plane curve contained in the plane $\Pi$. Choose a generic point $O^{\prime} \in \Pi, O^{\prime} \notin C$. The polar curve $C^{\prime}$ is defined as

$$
C_{O^{\prime}}^{\prime}=\operatorname{Pol}_{O^{\prime}}(C) \doteq\left\{\sum O_{i}^{\prime} \frac{\partial f}{\partial x_{i}}=0\right\}
$$

Note that $C \cap C^{\prime}=R_{C} \cup \operatorname{Sing}(C)$, where $R_{C}$ is the scheme of non-singular points $p \in C$ such that the tangent line to $C$ at $p$ contains $O^{\prime}$. The set $R_{C}$ depends on $O^{\prime}$, but the class $\left[R_{C}\right] \in A_{0}(C)$ is well defined. It follows that, scheme-theoretically,

$$
\left[R_{C}\right]=\left[C \cap C^{\prime}\right]-[\operatorname{Sing}(C)] .
$$

Lemma 3.3. We have the following equalities in $A_{0}(B)$ :

$$
\begin{aligned}
{[V]+[Q] } & =(v-2) h_{B}, \\
2[P]+3[Q]+\left[R_{B}\right] & =(v(v-1)-2 e-1) h_{B}=(d-1) h_{B}, \\
{\left[R_{B}\right] } & =(v-1) h_{B}-[V]-2[\text { Pinch }] .
\end{aligned}
$$

Proof. (3.3) follows from projecting the intersection $S^{\prime \prime} \cap B^{*}$ to $\mathbb{P}^{2}$ (see Lemma 2.9(3)), (3.4) follows by applying Definition 3.2 to $B$, and (3.5) is a new computation, follows from studying $B^{*} \cap S_{O^{\prime}}^{\prime}$. The multiplicities are found by a local computation, e.g., in [25, Chapter IX] or [22].

Subtract (3.5) from (3.4):

$$
2[P]+3[Q]-[V]-2[\mathrm{Pinch}]=(v(v-2)-2 e) h_{B} .
$$

From (3.3) we get $[Q]=(v-2) h_{B}-[V]$. Thus,

$$
2[P]+2[Q]-2[V]-2[\text { Pinch }]=((v-1)(v-2)-2 e) h_{B} .
$$

From the first equation in Lemma 3.1 we see that (by multiplying by 2) $2 e \cdot h_{B}=$ $2[\mathrm{Pinch}]+2[V]+2[V]+2[N]$ or $2[\mathrm{Pinch}]+2[V]=2 e h_{B}-2[V]-2[N]$. Substitute this in the equation above to get

$$
2[P]+2[Q]+2[V]+2[N]=((v-1)(v-2)) h_{B} .
$$

\subsection{Adjoint curves to the pure branch curve and to the double curve}

We now ask whether the reduced total branch curve $B \cup E$ has naturally defined adjoint curves.

Definition 3.4. Given a point $O^{\prime}$ as above, let $\left[e_{i}^{\vee}\right] \in A_{0}\left(E_{i}\right)$ denote the class of smooth points $p \in E_{i}$ such that the line $p O^{\prime}$ is tangent to $E_{i}$. This is the class corresponding to $R_{E_{i}}$ in Definition 3.2. 
We recall that Node is the set of nodes of $E$. We have the following equalities in the corresponding Chow groups:

\section{Lemma 3.5.}

$$
\begin{aligned}
{[V]+3[T] } & =(v-2) h_{E}, \\
{\left[V_{i}\right]+\sum_{j}\left[\tau_{i j}\right] } & =(v-2) h_{E_{i}}, \\
{\left[e^{\vee}\right]+2[\text { Node }]+6[T] } & =(e-1) h_{E}, \\
{\left[e_{i}^{\vee}\right]+\left.\sum[\text { Node }]\right|_{E_{i}}+2 \sum_{j}\left[\tau_{i j}\right] } & =(e-1) h_{E_{i}}, \\
{\left[e_{i}^{\vee}\right]+2\left[\text { Node }_{i}\right]+2 \sum_{j, \tau_{i j}>1}\left[\tau_{i j}\right] } & =\left(e_{i}-1\right) h_{E_{i}} .
\end{aligned}
$$

Proof. The first pair of equations is the projection of $\left[S^{\prime \prime} \cap E^{*}\right],\left[S^{\prime \prime} \cap E_{i}^{*}\right]$ (see Lemma 2.9). The next three equations come from $E \cap E^{\prime}, E_{i} \cap E^{\prime}$ and $E_{i} \cap E_{i}^{\prime}$.

\section{Lemma 3.6.}

$$
\left[\operatorname{Pinch}_{i}\right]=2\left[V_{i}\right]-2\left[e_{i}^{\vee}\right], \quad[\text { Pinch }]=2[V]-2\left[e^{\vee}\right] .
$$

Proof. Consider the locus in $\operatorname{Gr}(2,3) \times \operatorname{Gr}(2,3) \times \operatorname{Gr}(1,3)$ consisting of triples ( $\left.H, H^{\prime}, L\right)$ such that the line $L$ is contained in both planes. Then $V_{i}$ is the pullback to this locus of the class $\sigma_{1}$ in the first Grassmannian of planes, consisting of planes $H$ containing a given specified point $P, e_{i}^{\vee}$ is the pullback of the class $\sigma_{1}$ of lines $L$ meeting a given line $L^{\prime}$, and $\operatorname{Pinch}_{i}$ is the pullback of the diagonal in $\operatorname{Gr}(2,3) \times \operatorname{Gr}(2,3)$ where the planes $H$ and $H^{\prime}$ coincide. We can thus see that $2 e_{i}^{\vee}=\operatorname{Pinch}_{i}+2 V_{i}$ : the line $L$ meets one of two lines $L^{\prime}$ and $L^{\prime \prime}$ if and only if $L$ passes through one of the points where $L^{\prime}$ or $L^{\prime \prime}$ meets $H^{\prime}$. That is, either $H=H^{\prime}$ (and the class $\left(H, H^{\prime}, L\right)$ is in $\operatorname{Pinch}_{i}$ ), or $H$ contains one of these two points (and the class $\left(H, H^{\prime}, L\right)$ is in $2 V_{i}$ ).

Remark 3.7. This lemma implies that every component of $E$ contains vertical points. If $E_{i}$ is a component of $E$ with $e_{i}>1$, then $\left[e_{i}^{\vee}\right]=\left.\left[e^{\vee}\right]\right|_{E_{i}}>0$, so $2\left[V_{i}\right]=\left.2[V]\right|_{E_{i}}=$ $\left[\mathrm{Pinch}_{i}\right]+2\left[e_{i}^{\vee}\right]>0$. If $E_{i}$ is a line with no vertical points, then this means that no lines from the projection point $O$ to $E_{i}$ are tangent to the surface $S$. But the class of $\left[V_{i}\right]$ is invariant under the choice of generic $O$, so no lines from almost any point to $E_{i}$ are tangent to $S$. Hence $S$ must contain a plane containing $E_{i}$, which contradicts the hypothesis that $S$ is irreducible. Hence, if $E_{i}$ is a line, then it must still contain some $V_{i}$. Therefore, $B^{*} \cup F^{*}$ is connected, since every component of $F^{*}$ contains some points of $V^{*}$, but $V^{*}$ are intersection points of $F^{*}$ with $B^{*}$. Since $B^{*}$ is irreducible and every component of $F^{*}$ meets $B^{*}, B^{*} \cup F^{*}$ is connected.

Remark 3.8. Note that we now have equations for all the distinguished classes on $B \cup F$, i.e., for $[T],\left[\right.$ Node], [Pinch], $[V],[N],[Q],[P]$, in terms of the classes $h_{E}, h_{B}, e^{\vee}$ and $K_{E}$ :

$$
\begin{aligned}
6[T] & =2(v-2) h_{E}-\left([\text { Pinch }]+2\left[e^{\vee}\right]\right), \\
{[\text { Node }] } & =\frac{1}{2}(e-1) h_{E}-\left[e^{\vee}\right]-6[T],
\end{aligned}
$$




$$
\begin{aligned}
& {[V]=\frac{1}{2}\left([\text { Pinch }]+2\left[e^{\vee}\right]\right),} \\
& {[N]=e h_{B}-2[V]-[\text { Pinch }],} \\
& {[Q]=(v-2) h_{B}-[V],} \\
& {[P]=\frac{1}{2}(v-1)(v-2) h_{B}-[Q]-[V]-[N] .}
\end{aligned}
$$

Local versions also exist, of course, but are more cumbersome to write down. Note that these numerics agree with those that appear in [25].

3.2.1. Constructing some natural adjoint curves to $B+E$. We now construct some natural adjoint curves to the curve $B+E$ which later allow us to reconstruct the space curve $B^{*}+E^{*}$ from $B+E$.

In the classical Segre theory, in order to construct the natural adjoint curves, we start by computing the intersection of $B$ with its polar in $\mathbb{P}^{2}$ (see [8]). in $\mathbb{P}^{2}$.

In the singular case we compute the intersection of $B \cup F$ with the polar of $B \cup E$

Set-theoretically, the intersection is

$$
(B \cup F) \cap(B \cup E)^{\prime}=P \cup Q \cup \text { Node } \cup R_{B} \cup R_{E}
$$

(see Definition 3.2 for $R_{E}, R_{B}$ ).

Proposition 3.9. Scheme-theoretically, the intersection $(B \cup F) \cap(B \cup E)^{\prime}$ is

$$
2 P \cup 3 Q \cup 4 \text { Node } \cup 3 N \cup 3 \text { Pinch } \cup 6 V \cup 12 T \cup R_{B} \cup 2 R_{E} .
$$

Proof. We can see this from the following local computations:

- At $P$, the nodes of $B, B \cup F$ looks like $(x+y)(x-y)$, up to a change of local coordinates, and $(B \cup E)^{\prime}$ looks like $2 x$. The local intersection is the 2-dimensional vector space $\mathbb{C}\langle 1, y\rangle$.

- At $Q$, the cusps of $B, B \cup F$ looks like $x^{2}-y^{3}$, and $(B \cup E)^{\prime}$ looks like $2 x$. The local intersection is the 3-dimensional vector space $\mathbb{C}\left\langle 1, y, y^{2}\right\rangle$.

- At smooth points $R_{B}$ of $B$ where the tangent line passes through $0, B$ and $B^{\prime}$ meet transversely.

- At all other points of $B$, there is no intersection.

- At $N$ and Pinch, $B$ intersects $E$ transversely, so $B \cup F$ looks like $(x+y)(x-y)^{2}$, and $(B \cup E)^{\prime}$ looks like $2 x$. Hence, the local intersection is of multiplicity 3 .

- At $V, B$ is tangent to $E$, so $B \cup F$ looks like $\left((x-y)-(x+y)^{2}\right)(x-y)^{2}$, and $(B \cup E)^{\prime}$ looks like

$$
(x-y)(-2 x-1)+(x-y)-(x+y)^{2}=2 x(x-y)-(x+y)^{2}=x^{2}-4 x y+y^{2} .
$$

It vanishes to order 2 but is not tangent to either component of $B \cup F$, so the total multiplicity is 6 .

- At the triple points $T, B \cup F$ looks locally like $(x-y)^{2}(x+y)^{2}(x+2 y)^{2}$, and $(B \cup E)^{\prime}$ looks like $(x-y)(x+y)+(x-y)(x+2 y)+(x+y)(x+2 y)$, which vanishes to order 2 but has no common tangent directions with $B \cup F$, so the total multiplicity is 12 . 
- At the new nodes, Node, $B \cup F$ is locally $(x-y)^{2}(x+y)^{2}$, and $(B \cup E)^{\prime}$ is locally $2 x$, so the total intersection multiplicity is 4 .

- At the points $R_{E}=\left[e^{\vee}\right]$ where $E$ has a tangent line through $O^{\prime}, B \cup F$ passes twice and $(B \cup E)^{\prime}$ passes once, so the intersection multiplicity is 2 .

Thus, the polar $(B \cup E)^{\prime}$, which is of degree $v(v-1)-e-1$, intersects $B \cup F$ in

$$
2 P+3 Q+4 \text { Node }+3 N+3 \text { Pinch }+6 V+12 T+R_{B}+2 e^{\vee} .
$$

We recall the Residue Theorem (see e.g. Walker [26, Chap. VI, Theorem 6.2]):

Theorem 3.10 (Residue Theorem). Let $C$ be a plane curve. If $A, A^{\prime}, D$ are divisors on $C$ and $A$ and $A^{\prime}$ are linearly equivalent, and there is a curve $L$ intersecting $C$ in $A+D$, then there is also a curve $L^{\prime}$ of the same degree intersecting $C$ in $A^{\prime}+D$.

Note that the theorem is stated for $C$ a reduced curve and $D$ containing its adjoint divisor (Walker denotes our $D$ by $B+D$, where $D$ is the adjoint divisor and $B$ is some other positive divisor).

By the Residue Theorem, we can substitute $Q+3 V+6 T=(v-2) h_{B \cup F}$ (see equations (3.3) and (3.7)) to show that there exists a curve $W^{\prime}$ of degree $v(v-1)-e-1$ that intersects $B \cup F$ in

$$
2 P+2 Q+3 N+3 \text { Pinch }+3 V+6 T+4 \text { Node }+R_{B}+2 e^{\vee}+A_{v-2},
$$

where $A_{v-2}$ is the class of the intersection of a generic curve $C^{\prime}$ of degree $v-2$ with $B \cup F$ (explicitly, in the notation of the Residue Theorem, $A=Q+3 V+6 T, A^{\prime}=A_{v-2}$, $C=B \cup F)$. Since $\operatorname{deg} W^{\prime} \cdot \operatorname{deg} C^{\prime}<\operatorname{deg} A_{v-2}, C^{\prime}$ must be a component of $W^{\prime}$; hence $W^{\prime}$ must be reducible.

Explicitly, dropping the component of degree $v-2$, we are left with a curve of degree $v^{2}-2 v+1-e$ that meets $B \cup F$ in

$$
2 P+2 Q+3 N+3 \text { Pinch }+3 V+6 T+4 \text { Node }+R_{B}+2 e^{\vee} .
$$

We now apply the Residue Theorem again to replace equations (3.5), (3.12):

$$
(v-1) h_{B}=[V]+2[\text { Pinch }]+\left[R_{B}\right], \quad 2\left[e^{\vee}\right]+[\text { Pinch }]=2[V],
$$

to obtain a curve of degree $v^{2}-2 v+1-e$ that meets $B \cup F$ in

$$
2 P+2 Q+3 N+4 V+6 T+4 \text { Node }+A_{v-1}^{\prime},
$$

where $A_{v-1}^{\prime}$ is the class of the intersection of a generic curve of degree $v-1$ with $B$.

By adding to this curve a new component of degree $e$, we obtain a curve of degree $v^{2}-2 v+1$ that meets $B \cup F$ in

$$
2 P+2 Q+3 N+4 V+6 T+4 \text { Node }+A_{\nu-1}^{\prime}+A_{e},
$$

where $A_{e}$ is the class of the intersection of a generic curve of degree $e$ with $B \cup F$. Note that $\left[A_{e}\right]$ is $e h_{B \cup F}$ and thus is linearly equivalent to $v(v-1) h_{E}$, because both are linearly 
equivalent to the excess intersection class of $E \cap(B \cup F)$ in $A_{0}(E) \subset A_{0}(B \cup F)$. Using the fact that $(v-1) h_{B \cup F}=(v-1) h_{B}+2(v-1) h_{E}$, we can write

$$
\begin{aligned}
{\left[A_{v-1}^{\prime}\right]+\left[A_{e}\right] } & =(v-1) h_{B}+e h_{B \cup F}=(v-1) h_{B \cup F}+v(v-1) h_{E}-2(v-1) h_{E} \\
& =(v-1) h_{B \cup F}+(v-2)(v-1) h_{E} .
\end{aligned}
$$

Let $A_{v-1}, A_{(v-2)(v-1)}^{\prime \prime}$ be the classes of the intersection of a generic curve of degree $v-1$ (resp. $(v-2)(v-1))$ with $B \cup F$ (resp. $E$ ). Hence, there exists a curve of degree $v^{2}-2 v-1$ that meets $B \cup F$ in

$$
2 P+2 Q+3 N+4 V+6 T+4 \text { Node }+A_{\nu-1}+A_{(v-2)(v-1)}^{\prime \prime} .
$$

By degree considerations (as before), this curve must split into a component of degree $v-1$ and a component of degree $(v-1)(v-2)$ passing through

$$
2 P+2 Q+3 N+4 V+6 T+4 \text { Node }+A_{(v-2)(v-1)}^{\prime \prime} .
$$

Explicitly, in $A_{0}(B \cup F)$ we can write

$2 P+2 Q+3 N+4 V+6 T+4$ Node $+(v-2)(v-1) h_{E}=(v-2)(v-1) h_{B \cup F}$.

Subtracting (3.6) from the above equation we get

$$
2[V]+[N]+6[T]+4[\text { Node }]=(v-1)(v-2) h_{E} .
$$

Hence, applying the Residue Theorem one more time, there is a curve $L$ of degree $(v-1)(v-2)$ that intersects $B \cup F$ in

$$
2[P]+2[Q]+4[N]+6[V]+12[T]+8[\text { Node }] .
$$

Thus

$$
(v-1)(v-2) h_{B \cup F}=2[P]+2[Q]+4[N]+6[V]+8[\text { Node }]+12[T] .
$$

We recall the definition of an adjoint curve (see e.g. [11] for a classical treatment, or the modern definition in [1, Appendix A]):

Definition 3.11. Let $f(x, y)=0$ be the affine equation of a reduced plane curve $C \subset \mathbb{P}^{2}$ of degree $d$ with normalization $\phi: C^{*} \rightarrow C$. Let $p \in C$ be a singular point of $C$ and let $p_{1}, \ldots, p_{s}$ be the points of $C^{*}$ which lie over $p$. The adjoint divisor $\Delta_{p}$ of $p$ is the divisor on $C^{*}$ defined by $\Delta_{p}=\sum_{i}\left(a_{i} p_{i}\right)$ where $a_{i}=-\operatorname{mult}_{p_{i}}\left(\phi^{*} \frac{d x}{\partial f / \partial y}\right)$. For a plane curve with affine equation $g(x, y)=0$, define the zero divisor $\phi^{*}(g)$ to be the divisor of the meromorphic function $\phi^{*}(g)$ on $C$.

We say that a plane curve of affine equation $g(x, y)=0$ is adjoint to $f(x, y)=0$ at $p$ if $\phi^{*}(g) \geq \Delta_{p}$. Denoting $\Delta=\sum_{p \in \operatorname{Sing}(C)} \Delta_{p}$, a curve $A$ is adjoint to $C$ if $A \cdot C \geq \Delta$.

Note that the classical definition is the following: Given a plane curve $C$, another curve $A$ is said to be adjoint to $C$ if it contains each singular point of $C$ of multiplicity $r$ with multiplicity at least $r-1$. In particular, $A$ is adjoint to a nodal-cuspidal curve $C$ if it contains all nodes and all cusps of $C$. 
Definition 3.12. Denote for each singular point $p \in C_{\text {red }}, \Delta_{p}^{\prime}=0$ or $\sum_{i}\left(a_{i} p_{i}\right)$, and $\Delta^{\prime}=\sum_{p \in \operatorname{Sing}\left(C_{\text {red }}\right)} \Delta_{p}^{\prime}$. A curve $A$ is pseudo-adjoint to $C$ if $A \cdot C \geq \Delta^{\prime}$.

Proposition 3.13. There are two pseudo-adjoint curves $L, L_{1}$ to $B \cup F$ of degrees $(v-1)(v-2),(v-1)(v-2)+1$, passing through the points $P, Q, N, V$, Node and $T$ with intersection multiplicities $2,2,4,6,8$, and 12 , respectively.

Proof. Though not necessary for the proof, note that we have the following exact sequence:

$$
0 \rightarrow H^{0}(\Pi, \mathcal{O}((v-1)(v-2))) \stackrel{\text { res }}{\longrightarrow} H^{0}(B \cup F, \mathcal{O}((v-1)(v-2))) \rightarrow 0,
$$

as $\operatorname{deg}(B \cup F)=v(v-1)>(v-1)(v-2)$. Note that we could not have used this sequence to prove the existence of $L$, as we should have proved first that $2[P]+2[Q]+$ $4[N]+6[V]+8[$ Node $]+12[T]$ is a positive Cartier divisor.

Indeed, the existence of the adjoint curve $L$ of degree $(v-1)(v-2)$ was deduced above (see the discussion before (3.20)). The existence of a pseudo-adjoint curve $L_{1}$ of degree $(v-1)(v-2)+1$ follows from the fact that $B \cup F$ is a projection of a complete intersection curve in $\mathbb{P}^{3}$, by using $[8$, Corollary 4.24].

Remark 3.14. $L$ and $L_{1}$ are indeed pseudo-adjoint curves but not adjoint curves, as they do not pass through all the singular points of $B \cup F$ (e.g., the images Pinch of the pinch points).

Remark 3.15. Note that the curves $L$ and $L_{1}$ are also adjoint curves to $B$ of degrees $(v-1)(v-2),(v-1)(v-2)+1$.

\subsection{Possible tangent directions}

We need to determine the possible tangent directions of the pseudo-adjoint curves at the singular points of $B \cup F$ in order to see how these pseudo-adjoint curves reconstruct the space curve $B^{*} \cup F^{*}$. Explicitly, denote $L=\{f=0\}, L_{1}=\left\{f_{1}=0\right\}$. We want to identify the necessary restrictions on $L, L_{1}$ such that the $z$-coordinate of $B^{*} \cup F^{*}$ is given by $f_{1} / f$, i.e., the "new" singularities of $B \cup F$ are resolved by this definition of the $z$-coordinate.

Remark 3.16. By (3.19),

$$
2[V]+[N]+6[T]+4[\text { Node }]=(v-1)(v-2) h_{E},
$$

we see that the curve $L$ passes transversely to $E$ through the points $N$, and intersects it at $V$ (which are smooth points of $E$ ) with intersection multiplicity 2 . Therefore, $L$ must be tangent to $B$ at $N$ (so as to have intersection multiplicity 1 with $E$ and 2 with $B$ at $N$ ), must either have a node or be tangent to both curves at $V$, and must have nodes at $T$ and Node. 
To determine the possible tangent directions of $L$ and $L_{1}$, as in [8], we consider the set of Cartier divisors on $C=B \cup F$ passing through $\xi$, where $\xi=2[P]+2[Q]+$ $4[N]+6[V]+8[\mathrm{Node}]+12[T]$ is the 0 -cycle of the singularities to be resolved. We are interested in positive Cartier divisors $\zeta_{0}$ and $\zeta_{1}$, where $\zeta_{1}$ is of the form $\zeta_{1}=\zeta_{1}^{\xi}+\zeta_{1}^{\text {res }}$, $\zeta_{0}$ and $\zeta_{1}^{\xi}$ are supported on $\xi$, and $\zeta_{1}^{\text {res }}$ is supported on the smooth points of $C$. Note that the sections of the sheaf $\mathcal{O}_{C}\left(\zeta_{0}-\zeta_{1}\right)$ can locally be given by $r=h_{1} / h_{0}$, where $\operatorname{ord}_{p}(r)=\operatorname{ord}_{p}\left(h_{1}\right)-\operatorname{ord}_{p}\left(h_{0}\right) \geq 0$ at each singular point $p \in \xi$. As in [8], we have the adjunction sequence, for $a=(v-1)(v-2)$,

$$
\begin{aligned}
a_{C, i, \zeta_{1}}: J_{\zeta_{1}, \mathbb{P}}(a+i) & \stackrel{\operatorname{res}_{C}}{\longrightarrow} \mathcal{O}_{C}\left(-\zeta_{1}\right)(a+i) \stackrel{f_{l}^{a} / f_{L}}{\longrightarrow} \mathcal{O}_{C}\left(\zeta_{0}-\zeta_{1}\right)(i) \\
& =\mathcal{O}_{C}\left(\zeta_{0}-\zeta_{1}^{\xi}\right)(i)\left(-\zeta_{1}^{\mathrm{res}}\right) \subset \mathcal{O}_{C}\left(\zeta_{0}-\zeta_{1}^{\xi}\right)(i) \subset R_{C}(i)
\end{aligned}
$$

where the first map is restriction to $C$, the second map is induced from (3.20) (where $f_{l}, f_{L}$ are the equations of a line and the curve $L$, respectively), and $R_{C}$ is the sheaf of rational functions given locally by fractions $r=h_{1} / h_{0}$ such that $\operatorname{ord}_{Z}(r)=\operatorname{ord}_{Z}\left(h_{1}\right)-$ $\operatorname{ord}_{Z}\left(h_{0}\right) \geq 0$ for each codimension one subvariety $Z$ of $C$.

Remark 3.17 (Graded algebras for a space curve $C^{*}$ ). Assume we are given a space curve $C^{*}=B^{*} \cup F^{*}$ not contained in any plane in $\mathbb{P}^{3}$ and a projection $p: C^{*} \rightarrow$ $C$ to a plane curve $C$. Let $S=\bigoplus S_{i}, S_{i}=H^{0}(C, \mathcal{O}(i))$, be the graded algebra of homogeneous functions on $C$, and $T$ be the graded algebra of homogeneous functions on $C^{*}$. The inclusion $S \rightarrow T$ gives an isomorphism of fraction fields $\mathbb{Q}(S) \rightarrow \mathbb{Q}(T)$, since $C$ and $C^{*}$ are birational. Now $T_{1}=S_{1} \oplus k z$ for some element (the "vertical coordinate") $z \in T_{1}$; since $T_{1} \subset \mathbb{Q}(T) \simeq \mathbb{Q}(S)$, we should have

$$
z=f_{n+1} / f_{n}
$$

for some integer $n$ and plane curves $f_{n}$ and $f_{n+1}$ of degrees $n$ and $n+1$.

Corollary 3.18. Apply the previous remark to the space curve $C^{*}=B^{*} \cup F^{*}$, the generic projection $p: \mathbb{P}^{3} \rightarrow \mathbb{P}^{2}$ with center $O$ not on $C^{*}$ and the pseudo-adjoint curve L. Let $a=(v-1)(v-2)$.

Then the "vertical coordinate" $z$ on $C^{*}, z \in H^{0}\left(C^{*}, \mathcal{O}_{C^{*}}(1)\right)$, is the image of a uniquely defined plane curve $L_{1}$ of degree $a+1$ under the adjunction map $a_{C, 1}$ defined by (3.22).

In other words, we can choose $n=a$ in the remark above, and

$$
z=f_{L_{1}} / f_{L}
$$

where $f_{L}$ and $f_{L_{1}}$ are the equations of the plane curves $L$ and $L_{1}$, $\operatorname{deg} L_{1}=a+1$, and the curve $L_{1}$ is not a union of $L$ and a line, i.e., it is a "new" pseudo-adjoint curve. The curve $L_{1}$ must satisfy the following conditions:

(1) $L$ and $L_{1}$ meet $P$ and $Q$ transversely and have different tangent directions.

(2) $L$ and $L_{1}$ are both tangent to $B$ at $N$, and have intersection multiplicity 2 with each other. 
(3) $L$ and $L_{1}$ have nodes at $V$ such that one branch of $L$ is tangent to one branch of $L_{1}$, or either $L$ or $L_{1}$ is tangent to $B \cup E$ at $V$.

(4) $L$ and $L_{1}$ have nodes at $T$ with the same tangent cone, and their intersection multiplicity with each other is exactly 6.

(5) $L$ and $L_{1}$ have nodes at Node with distinct tangent cones.

Proof. Let $S$ be the graded homogeneous algebra of $C$, and $T$ be the graded homogeneous algebra of $C^{*}$; consider the element $t=z \cdot f_{L}$ of $T_{a+1}$. It is enough to prove that $t$ actually belongs to $S_{a+1}$, since then we can let $f_{a+1}=t$ and $z=f_{a+1} / f_{L}$. Now this is an easy local computation for each singular point of $C$, since the exact sequence

$$
0 \rightarrow S_{a+1} \rightarrow T_{a+1} \rightarrow T_{a+1} / S_{a+1} \rightarrow 0
$$

is obtained from the exact sequence of sheaves

$$
0 \rightarrow \mathcal{O}_{C}(a+1) \rightarrow p_{*} \mathcal{O}_{C^{*}}(a+1) \rightarrow \operatorname{Fac}(a+1) \rightarrow 0,
$$

where Fac is by definition the factor sheaf $p_{*} \mathcal{O}_{C^{*}} / \mathcal{O}_{C}$, by passing to global sections,

$$
0 \rightarrow H^{0}\left(C, \mathcal{O}_{C}(a+1)\right) \stackrel{p^{*}}{\rightarrow} H^{0}\left(C^{*}, \mathcal{O}_{C^{*}}(a+1)\right) \rightarrow \operatorname{coker} p^{*} \rightarrow 0 .
$$

Since the factor sheaf Fac is a product of sheaves supported at singular points of $C$, this makes computing the image of $t$ in $\left.H^{0}(C, \operatorname{Fac}(a+1))\right)$ an easy local computation at the singular points.

(1) The nodes $P$ and cusps $Q$ are resolved by a single blowup.

(2) A local model for $B \cup E$ at $N$ is $x y=0$, and since by intersection theory considerations $L$ is tangent to $B$, we can take $f_{L}=y-x^{2}$. Since $L$ vanishes to order 4 on $B \cup F$, so must $L_{1}$. Hence $L_{1}$ is tangent to $B$, say $f_{L_{1}}=y-2 x^{2}$. It is easy to check that the node is resolved and $t$ is in $S_{a+1}$.

(3) At $V, L$ and $L_{1}$ must either have nodes or be tangent to $B$. If they both have nodes at $V$, then suppose the tangent directions of $L$ are $v, w$ and the tangent directions of $L_{1}$ are $\alpha, \beta$. We want to blow up $V$ partially, to a transverse intersection. If $v=\alpha$ and $w=\beta$ then $V$ is not blown up at all. If $v \neq \alpha$ and $w \neq \beta$ then it blows up completely, unless one of the tangent directions is that of $B \cup E$. If $v$ is the tangent direction of $B \cup E$, or if $v=\alpha$ and $w \neq \beta$, then we have the following condition:

$$
\left(\frac{f_{L_{1}}}{f_{L}}\right)_{B^{*}}^{\prime}(t)=\left(\frac{f_{L_{1}}}{f_{L}}\right)_{F^{*}}^{\prime}(t),
$$

i.e., the intersection of $B^{*}$ and $F^{*}$ at $V^{*}$ is transverse.

For example a local model in the first case is

$V: y \cdot\left(y-x^{2}\right)=0, \quad L=\left\{\left(y+x^{2}\right)(y-2 x)=0\right\}, \quad L_{1}=\{(y-x)(y-3 x)=0\}$,

and a local model in the second case is

$$
V: y \cdot\left(y-x^{2}\right)=0, \quad L=\{x(y-2 x)=0\}, \quad L_{1}=\{(y-x)(y-2 x)=0\} .
$$


Another possibility is that $L$ is tangent at $V$ and $L_{1}$ has a node (or vice versa). Again,

$$
\left(\frac{f_{L_{1}}}{f_{L}}\right)_{B^{*}}^{\prime}(t)=\left(\frac{f_{L_{1}}}{f_{L}}\right)_{F^{*}}^{\prime}(t)
$$

as long as the tangent directions of $L_{1}$ are different from those of $L$ and $B \cup E$. A local model is

$$
V: y \cdot\left(y-x^{2}\right)=0, \quad L=\left\{y-2 x^{2}=0\right\}, \quad L_{1}=\{(y-x)(y-2 x)=0\} .
$$

(4) At $T, L$ and $L_{1}$ have nodes. If they have different tangent directions, then they will blow up $T$. To avoid blowing up, $L$ and $L_{1}$ must have the same tangent cone. A local model at $T$ is

$T: x \cdot y \cdot(y-x)=0, \quad L=\{(x-2 y)(x-3 y)=0\}, \quad L_{1}=\left\{(x-2 y)\left(x-3 y+x^{2}\right)=0\right\}$.

This model does not resolve the triple point but lifts one of the tangent directions out of the plane of the other two.

(5) $L$ and $L_{1}$ have nodes at [Node] by intersection theory considerations, and they resolve [Node] when the tangent cones of the two adjoint curves are different, e.g., $L=$ $\{(y-x)(y+x)=0\}, L_{1}=L=\{(y-2 x)(y+2 x)=0\}$.

Since $f_{L}$ vanishes at the singularities of $B \cup F$ that are resolved in $B^{*} \cup F^{*}, t=z f_{L}$ is a regular (holomorphic) object on $B \cup F$, and thus belongs to $S_{a+1}$.

\section{From adjoint curves to ramification curves}

Assume we are given a plane curve $C$ that consists of a reduced component $B$ and a double curve component $F$ (where $F_{\text {red }}=E$ ) such that $B$ has nodes $P$ and cusps $Q$, $E$ has nodes denoted by Node and triple points $T$, and that $B$ and $E$ intersect transversely (in points which we divide into two sets, called Pinch and $N$ ) and have simple tangencies at $V$. Suppose that these points satisfy all the numerical conditions of the previous section, and that the curve $C$ has pseudo-adjoint curves $L$ and $L_{1}$ satisfying the conditions of the previous section. In this section we prove that these conditions are sufficient for $C$ to be the (total) branch curve of a surface with ordinary singularities in $\mathbb{P}^{3}$.

Theorem 4.1. Let $C=B \cup F$ be a plane curve of degree $v(v-1)$ such that $B$ has nodes $P$ and cusps $Q$, and $F$ is a double curve such that $F_{\text {red }}=E$ is of degree e and has nodes Node and triple points $T$, and $B$ and $E$ intersect transversely in two distinguished sets of points, which are called the "pinch points" Pinch of the double curve $F$ and a set of "new nodes" $N$, and are simply tangent at a set of points $V$. The following conditions are necessary and sufficient for $C$ to be a (total) branch of a surface $S$ of degree $v$ with ordinary singularities:

(1) The distinguished points on $B, E$ satisfy the numerical conditions induced from (3.13)-(3.18) (see Remark 3.8).

(2) On each component of $E$ there is at least one point from the set $V$. 
(3) There are two pseudo-adjoint curves, L of degree $a=(v-1)(v-2)$ and $L_{1}$ of degree $a+1$, satisfying the constraints (1)-(5) of Corollary 3.18 on their intersections and tangent directions.

The necessity of these conditions was proved in Section 3. The following subsections provide the proof of the sufficiency.

\subsection{The model of the curve in $\mathbb{P}^{3}$}

We use the pseudo-adjoint curves to $B \cup F$ to construct a model of the curve $C$ in $\mathbb{P}^{3}$. Let $f_{L}$ be the equation of the curve $L$ and let $f_{L_{1}}$ be the equation of $L_{1}$. The equation $z=f_{L_{1}} / f_{L}$ defines a space curve $C^{*}=B^{*} \cup F^{*}$, whose projection to $\mathbb{P}^{2}$ is $C$. Note that $F^{*}$ really is a double space curve; its double structure is determined by using the differential of the function $z$ to lift the tangent vectors from the double structure of $F$.

Since $f_{L}$ is defined uniquely and $f_{L_{1}}$ is defined up to adding multiples of $f_{L}$, and since $z$ is defined by $f_{L}$ and $f_{L_{1}}$, the model $B^{*} \cup F^{*}$ is well defined up to coordinate changes generated by those of the form $(x: y: z: w) \mapsto(x: y: z+c: w)$; in particular, it is determined up to rational automorphisms of $\mathbb{P}^{3}$ that commute with projection to $\mathbb{P}^{2}$.

This model has the geometric properties of a ramification curve: by assumption it has all the correct numbers of distinguished points. It resolves each of the singularities correctly, by the calculations in Corollary 3.20.

\subsection{The model as a complete intersection}

We now prove the following theorem.

Theorem 4.2. The curve $B^{*} \cup F^{*}$ is a complete intersection.

First, we introduce the following definition:

Definition 4.3. Given a curve $C$, we define the index of speciality

$$
s(C)=\max \left\{n: h^{1}\left(C, \mathcal{O}_{C}(n)\right) \neq 0\right\} .
$$

(Note that many works denote the index by $e$ (see, e.g., Schlesinger [23]) but we call it $s$ to avoid confusion with the degree of $E$.)

To motivate our use of this definition we briefly recall some history. One of the first methods used to prove that an irreducible reduced space curve is a complete intersection of two surfaces of degrees $a$ and $b$ was Halphen's Speciality Theorem, introduced in 1882:

Theorem (see [14]). Let $C$ be a space curve of order $a \cdot b$ in $\mathbb{P}^{3}$ such that $a<b$ which has $\frac{1}{2} a(a-1) b(b-1)$ bisecants all lying on a cone of degree $(a-1)(b-1)$. Assume also that $C$ is not on a surface of degree smaller than $a$. Then $C$ is a complete intersection of two surfaces of degree $a$ and $b$. 
In the case of a smooth surface in $\mathbb{P}^{3}$, Segre used this method to show that a model built from a nodal-cuspidal plane curve is indeed a complete intersection (and later he showed that this space curve is an intersection of a surface and its polar). Indeed, every node and cusp are induced from a bisecant, so a space curve of degree $v(v-1)$, not on a surface of degree $v-2$, with nodes + cusps $=\frac{1}{2} v(v-1)^{2}(v-2)$ bisecants, lying on a cone of degree $(v-1)(v-2)$ (induced from the adjoint curve of this degree) is a complete intersection of two surfaces of degree $v$ and $v-1$.

However, as noted by Gruson and Peskine [13], the proof of Halphen involves "des considérations qui en rendent l'interprétation hasardeuse", and it is not at all clear whether it extends to the case of reducible or non-reduced curves. Gruson and Peskine rephrased this theorem in modern terms in 1978 as the Speciality Theorem:

Let $C$ be an integral curve in $\mathbb{P}^{3}$ of degree $d$, not contained in a surface of degree less than $t$. Let $s=s(C)$. Then $s \leq t+d / t-4$, with equality holding if and only if $C$ is $a$ complete intersection of type $(t, d / t)$ (and thus $\mathcal{O}_{C}(s)$ is special, i.e., $\left.h^{1}\left(\mathcal{O}_{C}(s)\right) \neq 0\right)$.

Substituting $t=v-1, d=v(v-1)$, we obtain Halphen's theorem.

However, in our case, the model of our curve in $\mathbb{P}^{3}$ is non-reduced and reducible - two phenomena that the Speciality Theorem does not address. However, in 1999, Schlesinger generalized the above theorem:

Theorem 4.4 ([23]). Let $C$ be a curve (possibly non-reduced and reducible) in $\mathbb{P}^{3}$ with index of speciality $s=s(C)$. Suppose that no subcurve $D$ of $C$ with $s(D)=s$ lies on a surface of degree $t-1$, and let $m$ be the minimum of $t$ and the integral part of $(s+4) / 2$. Then $\operatorname{deg} C \geq m(s+4-m)$ with equality holding if and only if $C$ is a deformation with constant cohomology of a complete intersection of two surfaces of degree $m$ and $s+4-m$.

Thus, in order to prove that $B^{*} \cup F^{*}$ is (a degeneration of) a complete intersection of surfaces of degrees $v$ and $v-1$ we have to prove that no subcurve of $B^{*} \cup F^{*}$ with the same index of speciality of $s\left(B^{*} \cup F^{*}\right)$ lies on a surface of degree less than $v-1$. We prove this in four steps.

Lemma 4.5. The index of speciality $s\left(B^{*} \cup F^{*}\right)$ is $2 v-5$, while all the subcurves of $B^{*} \cup F^{*}$, with the possible exception of $B^{*} \cup E^{*}$, have strictly lower speciality index.

Proof. In the case of $B^{*} \cup F^{*}$, the proof is the same as that of D'Almeida ([5] for the case of a smooth surface in $\left.\mathbb{P}^{3}\right)$ : by intersection theory considerations, we know that the curve $L$ does not meet $B \cup F$ outside the singular points. Hence there can be no curve of smaller degree containing the same set of singular points (counted with their multiplicities).

Let now $p: B^{*} \cup F^{*} \rightarrow B \cup F$ be the projection from the point $O$. The conductor of the structure sheaf $\mathcal{O}_{B^{*} \cup F^{*}}$ in $\mathcal{O}_{B \cup F}$ is $\operatorname{Ann}\left(p_{*} \mathcal{O}_{B^{*} \cup F^{*}} / \mathcal{O}_{B \cup F}\right)$, which by duality is isomorphic to $\operatorname{Ann}\left(\omega_{B \cup F} / p_{*}\left(\omega_{B^{*} \cup F^{*}}\right)\right)$ (see e.g. [2, Chapter 8]). By the definition of the conductor, we see that $\operatorname{Ann}\left(\omega_{B \cup F} / p_{*}\left(\omega_{B^{*} \cup F^{*}}\right)\right)=\operatorname{Hom}\left(\omega_{B \cup F}, p_{*}\left(\omega_{B^{*} \cup F^{*}}\right)\right)=$ $p_{*}\left(\omega_{B^{*} \cup F^{*}}\right) \otimes \omega_{B \cup F}^{\vee}$. It is well known that $H$ is a global section of the conductor sheaf iff $H$ passes through the singular points of the curve $B \cup F$ that get resolved. 
By Serre duality, for all $i, H^{1}\left(\mathcal{O}_{B^{*} \cup F^{*}}(i)\right)=H^{0}\left(\omega_{B^{*} \cup F^{*}}(-i)\right)$. Hence the minimal degree of a curve containing the points that get resolved when $B \cup F$ is lifted to $B^{*} \cup F^{*}$ is $v(v-1)-3-s\left(B^{*} \cup F^{*}\right)$. Setting this equal to $(v-1)(v-2)$, the degree of $L$, we see that $s\left(B^{*} \cup F^{*}\right)=2 v-5$.

We now examine the subcurves of $B^{*} \cup F^{*}$. Note that by our construction, the curve $B^{*} \cup F^{*}$ is connected, and $B^{*} \cap F^{*}$ consists only of $\mid$ Pinch $^{*}|+| V^{*} \mid$ (double) points.

- $s\left(B^{*}\right)$ : We use the fact that for any integral space curve $C$,

$$
2 p_{a}(C)-2 \geq \operatorname{deg}(C) s(C)
$$

(see, e.g., [16, Section 2]), as $2 p_{a}(C)-2-\operatorname{deg}(C) s(C)$ is the third Chern class of a rank two reflexive sheaf; this inequality is an equality in the case of subcanonical curves ([12]). (In the case of a smooth surface, the ramification curve is itself a complete intersection, hence subcanonical.) Suppose that $s\left(B^{*}\right)=2 v-5$, i.e. $2 p_{a}\left(B^{*}\right)-2 \geq$ $\operatorname{deg}\left(B^{*}\right)(2 v-5)$. Since

$$
p_{a}\left(B^{*}\right)=\frac{(v(v-1)-2 e-1)(v(v-1)-2 e-2)}{2}-n-c,
$$

we have

$$
(v(v-1)-2 e-1)(v(v-1)-2 e-2)-2 n-2 c-2 \geq(v(v-1)-2 e)(2 v-5) .
$$

Substituting for $n$ and $c$, we get

$$
\begin{aligned}
& (v(v-1)-2 e-1)(v(v-1)-2 e-2) \\
& \quad-v(v-1)(v-2)(v-3)+4 e(v-2)(v-3)+4\left(e^{v}\right)^{*}+24 t \\
& -4 e(e-1)-2 v(v-1)(v-2)+6 e(v-2)-6 t \\
& \quad \geq(v(v-1)-2 e)(2 v-5),
\end{aligned}
$$

which simplifies to $2+4\left(e^{\vee}\right)^{*}+18 T+12 e-6 v e \geq 0$. Since [Pinch $]=2[V]-2\left[e^{\vee}\right]$, we have $4\left[e^{\vee}\right]=4[V]-2[\mathrm{Pinch}]$. Hence this reduces to $2+4|V|-2|\operatorname{Pinch}|+18|T|+$ $6 e(2-v) \geq 0$. Since $e(v-2)=|V|+3|T|$, we get $2-2 \mid$ Pinch $|-2| V \mid \geq 0$, which is clearly impossible since $\mid$ Pinch $+V \mid$ is always greater than 1 .

- $s\left(F^{*}\right)$ : Consider two Cohen-Macaulay curves $Y_{1}, Y_{2} \subseteq \mathbb{P}^{3}$ meeting in a zero-dimensional subscheme $Z^{*}$, let $Y=Y_{1} \cup Y_{2}, Z^{*}=Y_{1} \cap Y_{2}$. Then we have the following exact sequence (see $[15$, p. 82$])$ :

$$
0 \rightarrow \omega_{Y} \rightarrow \omega_{Y_{1}}\left(Z^{*}\right) \oplus \omega_{Y_{2}}\left(Z^{*}\right) \rightarrow \mathbb{C}^{\oplus Z^{*}} \rightarrow 0 .
$$

Twisting by $-k$ and taking cohomology, we see that

$$
h^{0}\left(\omega_{Y}(-k)\right)=h^{0}\left(\omega_{Y_{1}}\left(-k h_{Y_{1}}+Z^{*}\right)\right)+h^{0}\left(\omega_{Y_{2}}\left(-k h_{Y_{2}}+Z^{*}\right)\right) .
$$

Hence, if $Z^{*}$ is at least equal to $h_{Y_{1}}$ (in the partial order of the Chow group $A_{0}(Y)$ ), then $s\left(Y_{1}\right)<s(Y)$, a strict inequality. In our case, since $B^{*}$ is irreducible, we can divide 
$B^{*} \cup F^{*}$ into two components as $B^{*}$ and $F^{*}$. The intersection $[Z]^{*}$ is $[\mathrm{Pinch}]^{*}+[V]^{*}$. We need to prove that $\left[Z^{*}\right]>h_{F^{*}}=2 h_{E^{*}}$. Denote by $Z$ the image of $Z^{*}$ in $\mathbb{P}^{2}$; it is enough to show that $[Z]=[$ Pinch $]+[V]>2 h_{E}$.

We know that $[V]+3[T]=(v-2) h_{E}$, and that $0<[$ Pinch $]=2(v-4) h_{E}-2 C_{E}-$ $6[T]$, so $3[T]<(v-4) h_{E}$. Hence $[V]$ must be at least $2 h_{E}$, or $[Z]=[$ Pinch $]+[V]$ $>2 h_{E}$. Hence $s\left(F^{*}\right)<s\left(B^{*} \cup F^{*}\right)$.

Let $F_{1}^{*}$ be a component of $F^{*}$.

- $s\left(B^{*} \cup F_{1}^{*}\right)$ : For $T=0$, assume $s\left(B^{*} \cup F_{1}^{*}\right)=2 v-5$. The minimal degree of a curve passing through all the singularities of $B \cup F_{1}$ that should be resolved must be

$$
\left(v(v-1)-2 e+2 e_{1}\right)-3-(2 v-5)=(v-1)(v-2)-2 e+2 e_{1} \doteq b
$$

(by the same reasoning as in the case of $B^{*} \cup F^{*}$ ). Assume such a curve $C_{0}$ of degree $b$ exists. Then

$$
C_{0} \cap\left(B \cup F_{1}\right)=2 P+2 Q+4 N_{1}+6 V_{1}+8 \text { Node }_{1}+\{\text { Residual points },
$$

by intersection theory considerations. But

$$
\begin{aligned}
b \cdot \operatorname{deg}\left(B \cup F_{1}\right) & =\left((v-1)(v-2)-2 e+2 e_{1}\right) \operatorname{deg}\left(B \cup F_{1}\right) \\
& =2 P+2 Q+4 N_{1}+6 V_{1}+8 \operatorname{Node}_{1}-2\left(e-e_{1}\right) \operatorname{deg}\left(B \cup F_{1}\right)
\end{aligned}
$$

(by (3.20) restricted to $B \cup F_{1}$ ). So we should get

$$
b \cdot \operatorname{deg}\left(B \cup F_{1}\right)=\left|C_{0} \cap\left(B \cup F_{1}\right)\right|,
$$

or

$$
-2\left(e-e_{1}\right) \operatorname{deg}\left(B \cup F_{1}\right)=\{\text { Residual points }\},
$$

which is a contradiction.

However, if such a curve does not exist, then the degree of the minimal-degree curve is $\operatorname{not} b=(v-1)(v-2)-2 e+2 e_{1}$, i.e., $s\left(B^{*} \cup F^{*}\right)<2 v-5$. Likewise, for $T>0$, the minimal degree of a curve passing through the singularities is still $\left(v(v-1)-2 e+2 e_{1}\right)$ $-3-(2 v-5)=(v-1)(v-2)-2 e+2 e_{1}=b$. Assume that such a curve $C_{0}$ exists. Then

$$
C_{0} \cap\left(B \cup F_{1}\right)=2 P+2 Q+4 N_{1}+6 V_{1}+8 \text { Node }_{1}+3 T_{1}+\{\text { Residual points }\},
$$

where $T_{1}$ denotes the triple points that are triple points of $F_{1}$. (The other triple points appear as nodes or smooth points of $F_{1}$ and hence do not have to be resolved.) However,

$$
\begin{aligned}
& b \cdot \operatorname{deg}\left(B \cup F_{1}\right)=\left((v-1)(v-2)-2 e+2 e_{1}\right) \operatorname{deg}\left(B \cup F_{1}\right) \\
& \quad=2|P|+2|Q|+4\left|N_{1}\right|+6\left|V_{1}\right|+8\left|\operatorname{Node}_{1}\right|+\sum_{j}\left|\tau_{1 j}\right|-2\left(e-e_{1}\right) \operatorname{deg}\left(B \cup F_{1}\right),
\end{aligned}
$$

so

$$
b \cdot \operatorname{deg}\left(B \cup F_{1}\right)=\left|C \cap\left(B \cup F_{1}\right)\right|+\sum_{j,\left|\tau_{1 j}\right| \neq 3}\left|\tau_{1 j}\right| .
$$


Hence

$$
-2\left(e-e_{1}\right) \operatorname{deg}\left(B \cup F_{1}\right)=\{\text { Residual points }\}-\sum_{j,\left|\tau_{1 j}\right| \neq 3}\left|\tau_{1 j}\right| .
$$

By (3.8), $V_{2}+\sum_{j} \tau_{2 j}=(v-2) e_{2}$, so $\sum_{j} \tau_{2 j}<(v-2) e_{2}$. Also

$$
\sum_{j,\left|\tau_{1 j}\right| \neq 3} \tau_{1 j} \leq 2 \sum_{j, \tau_{2 j} \neq 3} \tau_{2 j} \leq 2(v-2) e_{2}
$$

Hence

$$
\begin{aligned}
\{\text { Residual points }\} & <-2\left(e-e_{1}\right) \operatorname{deg}\left(B \cup F_{1}\right)+\left(e-e_{1}\right)(2 v-4) \\
& =\left(e-e_{1}\right)\left(2 v-4-2\left(v(v-1)-2 e+2 e_{1}\right)\right) \\
& =\left(e-e_{1}\right)\left(-2 v^{2}+4 v-4+4 e-4 e_{1}\right) \\
& <\left(e-e_{1}\right)\left(-2 v^{2}+4 v-4+2(v-1)(v-2)\right) \\
& =\left(e-e_{1}\right)(-2 v)<0 .
\end{aligned}
$$

This is a contradiction, as above, so the degree of the minimal curve cannot be $b$, and hence $s\left(B^{*} \cup F_{1}^{*}\right) \neq 2 v-5$.

- As $\max \left(s\left(B^{*}\right), s\left(F_{i}^{*}\right)\right) \leq s\left(B^{*} \cup F_{i}^{*}\right)$, it follows that $s\left(F_{i}^{*}\right)<2 v-5$.

- As for the subcurves $E_{i}^{*}$ of $F_{i}^{*}$ (and likewise $B^{*} \cup E_{i}^{*}$ ), consider the exact sequence

$$
0 \rightarrow \mathcal{I}_{E}(k) \rightarrow \mathcal{O}_{F}(k) \rightarrow \mathcal{O}_{E}(k) \rightarrow 0
$$

The last terms of the long exact sequence of cohomology are

$$
H^{1}\left(\mathcal{O}_{F}(k)\right) \rightarrow H^{1}\left(\mathcal{O}_{E}(k)\right) \rightarrow 0
$$

Thus $s(F) \geq s(E)$.

We next verify that the curve $B^{*} \cup F^{*}$ does not lie on a surface of degree lower than the degree of the polar of the surface we wish to construct.

Lemma 4.6. The curve $B^{*} \cup E^{*}$ does not lie on a surface of degree less than $v-1$.

Proof. Let $S$ be a surface containing $B^{*} \cup E^{*}$. Since $B^{*}$ is smooth at $Q$ while $B$ has a cusp at $Q, B^{*}$ and $E^{*}$ are transverse at $V^{*}$ while $B$ and $E$ are tangent, and $E^{*}$ has a non-planar triple point at $T^{*}$ while $E$ has a planar triple point at $T$, the projection to $\mathbb{P}^{2}$ kills an element of the tangent space to $B^{*} \cup E^{*}$, and hence of the tangent space to $S$, at each of these points. Therefore, the polar to $S$ contains $Q^{*}, V^{*}$ and $T^{*}$. Thus

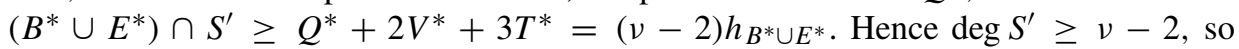
$\operatorname{deg} S \geq v-1$.

These two lemmas satisfy the premises of Schlesinger's generalized Speciality Theorem (Theorem 4.4), which we can apply to conclude that $B^{*} \cup F^{*}$ is a degeneration of a complete intersection. 
Lemma 4.7. The curve $B^{*} \cup F^{*}$ is a degeneration of complete intersections of surfaces of degrees $v$ and $v-1$, preserving the dimensions of the cohomologies of the twisted ideal sheaves.

Proof. By Theorem 4.4, it is enough to check that no subcurve $C$ of $B^{*} \cup F^{*}$ with the same index of speciality $s\left(B^{*} \cup F^{*}\right)$ lies on a surface of lower degree.

The speciality of $B^{*} \cup F^{*}$ is $2 v-5$, by Lemma 4.6. $B^{*} \cup E^{*}$ cannot lie on a surface of degree less than $v-1$, and $B^{*} \cup F^{*}$ clearly cannot lie on any surface that does not contain its subcurve $B^{*} \cup E^{*}$. Finally, all the other subcurves of $B^{*} \cup F^{*}$ have speciality index strictly lower than $2 v-5$, as was shown in Lemma 4.5.

In fact, our curve is not only a degeneration of complete intersections, but is itself a complete intersection.

Theorem 4.8. The curve $B^{*} \cup F^{*}$ is itself a complete intersection of two surfaces of degrees $v$ and $v-1$.

Proof. A complete intersection of surfaces of degrees $v$ and $v-1$ lies on one surface of degree $v-1$ and a 4-dimensional family of surfaces of degree $v$, generated by the products of three linear forms with the form of degree $v-1$ and one independent degree $v$ form. Since the degeneration is required to be cohomology-preserving, it follows that the limit $B^{*} \cup 2 E^{*}$ also lies on a surface of degree $v-1$ and a 4-dimensional family of surfaces of degree $v$. Hence it lies on the intersection of a surface $\Sigma$ of degree $v-1$ and an independent surface $S$ of degree $v$. Since the degrees match, the intersection is complete.

\subsection{The complete intersection of a surface and its polar}

We can now prove our main theorem.

Proof of Theorem 4.1. Consider the 5-dimensional vector space $W$ of $v$-forms $S_{t}$ of the form $t_{0} S+t_{1} x F+t_{2} y F+t_{3} z F+t_{4} w F$. The generic $S_{t}$ is a smooth surface, whose intersection with its polar is the ramification curve of $S_{t}$. The intersection of $B^{*}$ with $S_{t} \cap S_{t}^{\prime}$ includes $Q^{*}$ and $V^{*}$, since a tangent direction of the curve $B^{*} \cup 2 E^{*}$ gets collapsed at these points, so any surface containing $B^{*} \cup 2 E^{*}$ must have a vertical tangent direction at these points. But by degree considerations, $\left[B^{*} \cap S_{t} \cap S_{t}^{\prime}\right]=(v-1) h_{B^{*}}$, whereas $\left[Q^{*}\right]+\left[V^{*}\right]=(v-2) h_{B^{*}}$. Hence $B^{*} \cap S_{t} \cap S_{t}^{\prime}=Q^{*}+V^{*}+r_{B^{*}}(t)$, where $r_{B^{*}}(t) \in$ $\left|h_{B^{*}}\right|$. This $r_{B^{*}}(t)$ thus determines (at least generically) a linear map from $W$ to the 4dimensional vector space $H^{0}\left(\mathcal{O}_{B^{*}}(1)\right)=H^{0}\left(\mathcal{O}_{\mathbb{P}^{3}}(1)\right)$, by sending $S_{t}$ to $|t| \phi\left(r_{B^{*}}(t)\right)$, where $|t|$ is the magnitude of the vector $\left(t_{0}, \ldots, t_{4}\right)$ and $\phi\left(r_{B^{*}}(t)\right)$ is the normalized 1form that vanishes on $r_{B^{*}}(t)$.

Likewise, the intersection of $F^{*}$ with $S_{t} \cap S_{t}^{\prime}$ includes $2 V^{*}+6 T^{*}$, since vertical tangent directions get collapsed and the local intersection of $F^{*}$ with any other curve is at least 2 at $V^{*}$ and at least 6 at $T^{*}$. Since $\left[2 V^{*}\right]+\left[6 T^{*}\right] \in(v-2) h_{F^{*}}$, and $\left[F^{*} \cap S_{t} \cap S_{t}^{\prime}\right] \in$ $(v-1) h_{F^{*}}$, we find that $F^{*} \cap S_{t} \cap S_{t}^{\prime}=2 V^{*}+6 T^{*}+r_{F^{*}}(t)$, where $r_{F^{*}}(t) \in\left|h_{F^{*}}\right|$. Thus we get another generic map from $W$ to $H^{0}\left(\mathcal{O}_{\mathbb{P}^{3}}(1)\right)$. Finally, if we play the same game with $B^{*} \cup F^{*}$, then $r_{B^{*}}(t)+r_{F^{*}}(t) \in\left|h_{B^{*} \cup F^{*}}\right|$ determines yet another map to $H^{0}\left(\mathcal{O}_{\mathbb{P}^{3}}(1)\right)$. 
Any map from a 5-dimensional vector space to a 4-dimensional one has at least a one-dimensional kernel. The only way such a kernel can arise is when the class $r(t)$ is not well defined, i.e., if the intersection of the curves is an excess intersection rather than a set of points including $Q^{*}, V^{*}$ and $T^{*}$, i.e., if $B^{*}$ or $F^{*}$ is a component of the ramification curve. Hence the kernel of $r_{B^{*}}(t)$ is the linear space of $\nu$-forms that define surfaces whose ramification curve contains $B^{*}$, and the kernel of $r_{F^{*}}(t)$ consists of $v$-forms defining surfaces whose ramification curve contains the double curve $F^{*}$. The kernel of the third map $r_{B^{*} \cup F^{*}}$ is the set of $\nu$-forms for which either $B^{*}$ or $F^{*}$ is in the ramification curve. Since the kernel of a linear map is a linear subspace, not a union of linear subspaces, we conclude that the above two kernels coincide; that is, there exists a surface $S_{t}$ such that $S_{t} \cap S_{t}^{\prime}$ contains $B^{*} \cup F^{*}$. By degree considerations, $S_{t} \cap S_{t}^{\prime}=B^{*} \cup F^{*}$.

Hence $B^{*} \cup F^{*}$ is the intersection of a surface and its polar; in other words, it is a ramification curve.

Remark 4.9. Note that the surface $S_{t}$ whose ramification curve is $B+F$ is uniquely defined by the model $B^{*}+F^{*}$, since $B^{*}+F^{*}$ can only lie on a single surface $S_{t}^{\prime}$, and $S_{t}$ is determined by its polar up to translation along the $z$-axis. Thus the surface, like its ramification curve, is uniquely determined by $B+F$ up to automorphisms of $\mathbb{P}^{3}$ that commute with projection.

The construction in this paper thus provides a constructive proof of an analogue of Chisini's theorem for surfaces with ordinary singularities in $\mathbb{P}^{3}$. In particular, given the total branch curve of such a surface, one can construct its model in $\mathbb{P}^{3}$ from the pseudoadjoint curves, which are determined by the special divisor class of $B \cup F$. This model is then a complete intersection of a surface $S$ and its polar. This $S$ is the original surface, up to changes of coordinates that commute with projection.

\section{Examples}

We first introduce some notation.

Notation 5.1. Let $V(c, d, n)$ (resp. $B(c, d, n)$ ) be the variety of degree $d$ plane curves (resp. branch curves of generic linear projections) with $c$ cusps and $n$ nodes.

By Remark 3.15, equation (3.6) and the exact sequence (3.21), there exists a curve $L$ of degree $(v-1)(v-2)$ that passes (smoothly) through the cusps $Q$ and the nodes $P$ of the (pure) branch curve $B$, is tangent to $B$ at the points $N$ and has nodes at the points $V$. The curve $L$ is unique if the exact sequence (3.21) is exact for $B$ as well as for $B \cup F$, in particular if $d>(v-1)(v-2)$. Therefore, in this case

$$
\{L\} \simeq H^{0}\left(\mathbb{P}^{2}, J_{\zeta}(a)\right)
$$

where $\zeta=P+Q+2 V+2 N$ is a Cartier divisor on $B$. Note that $2 N$ is a summand of $\zeta$, as $L$ has a specified tangent direction at this point, and to pass through a point with a given tangent direction is equivalent to passing through two points (the actual point and one infinitely near point). 
Let $\xi$ be a 0 -cycle in $\mathbb{P}^{2}$. Define the superabundance of $\xi$ (relative to degree $n$ curves) as

$$
\delta(\xi, n)=h^{1} J_{\xi}(n)
$$

We first examine the case of a surface with a double line.

\subsection{Surfaces with a double line}

A surface $S \subset \mathbb{P}^{3}$ of degree $v$ with a double line as its only double curve has the following invariants:

$$
\begin{gathered}
d=\operatorname{deg}(B)=v(v-1)-2, \quad e=\operatorname{deg}(E)=1, \quad c=v(v-1)(v-2)-3(v-2), \\
n=\frac{1}{2} v(v-1)(v-2)(v-3)-2(v-2)(v-3), \quad p=\mid \text { Pinch }|=2(v-2), \quad| V \mid=v-2, \\
|N|=(v-2)(v-3), \quad \mid \text { Node } \mid=0, \quad t=0, \quad e_{1}=0 .
\end{gathered}
$$

These numerics are classical; the history is explained in some detail by Ragni Piene [22].

Set $\zeta=P+Q+2 V+2 N$ and $a=(v-1)(v-2)$. Note that in this case $d>a$.

Proposition 5.2 (Speciality index of $\zeta$ ). The speciality index of $\zeta$ satisfies

$$
\delta(\zeta, a)=\frac{1}{2}(v-2)(v-3)(2 v-1) .
$$

Proof. For the expected dimension $\operatorname{vdim}\left|J_{\zeta}(a)\right|$, we have

$$
\operatorname{vdim}\left|J_{\zeta}(a)\right|=\operatorname{dim}|a h|-\operatorname{deg} \zeta=\frac{1}{2} a(a+3)-\frac{1}{2}(v-2)^{2}\left(v^{2}+1\right) .
$$

Since $a=(v-1)(v-2)$, we get

$$
\operatorname{vdim}\left|J_{\zeta}(a)\right|=\frac{1}{2}(v-2)\left(-2 v^{2}+7 v-3\right)
$$

Since, by the definition of the speciality index,

$$
\operatorname{dim}\left|J_{\zeta}(d)\right|=\operatorname{vdim}\left|J_{\zeta}(d)\right|+\delta(\zeta, d),
$$

and since $\left|J_{\zeta}(a)\right|=\{L\}$, we get the equality. Likewise, $\delta(\zeta, a+1)$ can be computed analogously.

Thus, $\delta(\zeta, a)>0$ only when $v>3$. For example, for $v=4, \delta(\zeta, 6)=7$. Indeed, the curve $L$ (which is adjoint to $B \in B(10,18,8)$ ) passes through 34 points, counted with multiplicity. However, it is not known if there is a curve $C \in V(10,18,8)$ such that $\delta(\zeta, 6)<7$. 


\subsection{Degree 4 surfaces}

As was noted at the end of the last subsection, for a degree 4 surface in $\mathbb{P}^{3}$ with a double line, the 0 -cycle $\zeta=P+Q+2 V+2 N$ is special, and $\delta(\zeta, 6)=7$. We will now look at other degree 4 surfaces in $\mathbb{P}^{3}$ such that this 0 -cycle is special with respect to degree 6 curves passing through it (as $L$ is a degree 6 curve passing through $\zeta$ ).

(1) Double curve is a smooth conic: In this case, $e=\operatorname{deg}(E)=2, d=\operatorname{deg}(B)=8$, $|Q|=12,|P|=4,|N|=4,|V|=4$. Thus $\operatorname{deg} \zeta=32$ and $\delta(\zeta, 6)=5$. The variety $V(8,12,4)$ is in fact reducible; there is a Zariski pair for this curve (see [7]).

(2) Double curve is a union of two skew lines: In this case, $e=2, d=8,|Q|=12$, $|P|=8,|N|=0,|V|=4$. Thus $\operatorname{deg} \zeta=28$ and $\delta(\zeta, 6)=1$. The variety $V(8,12,8)$ is irreducible, i.e., for every curve $C \in V(8,12,8)$ the 0 -cycle $\zeta$ is special with respect to degree 6 curves.

(3) Double curve is a rational space cubic or a union of three lines meeting in a point: In these two cases, $d=a$ and it is not known whether $\zeta$ is a special 0 -cycle.

Acknowledgments. The authors wish to thank Luca Chiantini, Christian Peskine, Ragni Piene, Alessandra Trambaiolli, Enrico Schlesinger and Jonathan Wahl, among others, for many stimulating discussions.

This work is partially supported by the Emmy Noether Research Institute for Mathematics (center of the Minerva Foundation of Germany), the Excellency Center "Group Theoretic Methods in the Study of Algebraic Varieties" of the Israel Science Foundation.

The first author would like to thank the Max Planck Institute at Bonn for their warm hospitality, which considerably helped to finalize this work.

\section{References}

[1] Arbarello, E., Cornalba, M.: Griffiths, P. A.: Harris, J., Geometry of Algebraic Curves. Vol. 1, Springer (1984) Zbl 0559.14017 MR 0770932

[2] Altman, A., Kleiman, S.: Introduction to Grothendieck Duality Theory. Springer (1970) Zbl 0215.37201 MR 0274461

[3] Chisini, O.: Sulla identita birazionale delle funzioni algebriche di due variabili dotate di una medesima curva di diramazione. Rend. Ist. Lombardo 77, 339-356 (1944) Zbl 0061.35305 MR 0019351

[4] Ciliberto, C., Flamini, F.: On the branch curve of a general projection of a surface to a plane. Trans. Amer. Math. Soc. 363, 3457-3471 (2011) Zbl pre05934519 MR 2775814

[5] D'Almeida, J.: Courbe de ramification de la projection sur $\mathbb{P}^{2}$ d'une surface de $\mathbb{P}^{3}$. Duke Math. J. 65, 229-233 (1992) Zbl 0766.14018 MR 1150585

[6] Franchetta, A.: Sulla curva doppia proiezione di una superficie generale dell' $S_{4}$, da un punto generico su un $S_{3}$. Rend. Accad. Italia (7) 2, 282-288 (1941) and Rend. Lincei Sci. Fis. Mat. Nat. 2, 276-279 (1947) Zbl 0025.36001(1941) Zbl 0029.31601(1947) MR 0017949(1941) MR 0022696(1947)

[7] Friedman, M., Teicher, M.: New restrictions for branch curves and new Zariski pairs. Submitted

[8] Friedman, M., Leyenson, M.: On ramified covers of the projective plane I: Segre's theory and classification in small degrees (with an appendix by Eugenii Shustin). Int. J. Math. 22, 619-653 (2011) Zbl pre05912168 MR 2799882 
[9] Fulton, W.: Intersection Theory. Springer (1998) Zbl 0885.14002 MR 1644323

[10] Fulton, W., Hansen, J.: A connectedness theorem for projective varieties, with applications to intersections and singularities of mappings. Ann. of Math. (2) 110, 159-166 (1979) Zbl 0389.14002 MR 0541334

[11] Greco, S., Valabrega, P.: On the theory of adjoints. In: Algebraic Geometry, K. Lønsted (ed.), Lecture Notes in Math. 732, Springer, 98-123 (1979) Zbl 0408.14002 MR 0555694

[12] Griffiths, P., Harris, J.: Principles of Algebraic Geometry. Wiley, New York (1978) Zbl 0408.14001 MR 0507725

[13] Gruson, L., Peskine, C.: Genre des courbes de l'espace projectif. In: Algebraic Geometry (Troms $\varnothing$, 1977), Lecture Notes in Math. 687, Springer, 31-59 (1978) Zbl 0412.14011 MR 0527229

[14] Halphen, G. H.: Mémoire sur la classification des courbes gauches algébriques. J. École Polytechnique 52, 1-200 (1882)

[15] Harris, J., Morrison, I.: Moduli of Curves. Springer, New York (1998) Zbl 0913.14005 MR 1631825

[16] Hartshorne, R.: Stable reflexive sheaves. Math. Ann. 254, 121-176 (1980) Zbl 0431.14004 MR 0597077

[17] Kulikov, V.: On Chisini's conjecture. Izv. Ross. Akad. Nauk Ser. Mat. 63, no. 6, 83-116 (1999) (in Russian) Zbl 0962.14005 MR 1748562

[18] Kulikov, V.: On a conjecture of Chisini for coverings of the plane with A-D-E-singularities. In: Real and Complex Singularities, Lecture Notes in Pure Appl. Math. 232, Dekker, New York, 175-188 (2003) Zbl 1081.14050 MR 2075064

[19] Kulikov, V.: On characterization of branch curves of generic coverings. In: Mathematics: Fundamental Problems, Applications, Teaching, Vol. 2, Moscow State Univ. Publ., 66-102 (2002)

[20] Kulikov, V.: On Chisini's conjecture II. Izv. Ross. Akad. Nauk Ser. Mat. 72, no. 5, 63-76 (2008) (in Russian) Zbl 1153.14012 MR 2473772

[21] Moishezon, B.: Complex Surfaces and Connected Sums of Complex Projective Planes. Lecture Notes in Math. 603, Springer, New York (1977) Zbl 0392.32015 MR 0491730

[22] Piene, R.: Some formulas for a surface in $\mathbb{P}^{3}$. In: Algebraic Geometry (Troms $\left.\varnothing, 1977\right)$, Lecture Notes in Math. 687, Springer, 196-235 (1978) Zbl 0391.14008 MR 0527235

[23] Schlesinger, E.: A speciality theorem for Cohen-Macaulay space curves. Trans. Amer. Math. Soc. 351, 2731-2743 (1999) Zbl 0922.14020 MR 1641111

[24] Segre, B.: Sulla caratterizzazione delle curve di diramazione dei piani multipli generali. Mem. Accad. Italia 1, no. 4, 31 pp. (1930) Zbl 56.0562.01

[25] Semple, J., Roth, L.: Introduction to Algebraic Geometry. Oxford Univ. Press (1949) Zbl 0041.27903 MR 0034048

[26] Walker, R. J.: Algebraic Curves. Springer (1978) Zbl 0399.14016 MR 0513824

[27] Zariski, O.: On the problem of existence of algebraic functions of two variables possessing a given branch curve. Amer. J. Math. 51, 305-328 (1929) Zbl 55.0806.01 MR 1506719 